\title{
Development of a Method for Determining the Dependence of the Electron Mobility on the Longitudinal-Electric Field in MOSFETs
}

\author{
J. B. ROLDÁN *, F. GÁMIZ and J. A. LÓPEZ-VILLANUEVA \\ Departamento de Electrónica y Tecnología de Computadores, Universidad de Granada, \\ Facultad de Ciencias, Avd., Fuentenueva s/n, 18071 Granada Spain
}

\begin{abstract}
A new experimental method for determining the dependence of the electron mobility on the longitudinal-electric field has been developed. The development, validation and explanation of this new method has been carefully carried out. We have applied this procedure to standard submicron MOSFETs and after having obtained the mobility dependence on both the transverse- and longitudinal-electric fields we reproduced the experimental output curves. The saturation velocity has also been calculated using the mobility curves obtained by this new method.
\end{abstract}

Keywords: Electron mobility, longitudinal-electric field, saturation velocity, output curves, driftdiffusion simulation

\section{INTRODUCTION}

The electron-mobility dependence on the longitudinal-electric field, oriented along the channel, has been studied experimentally. A "closed-loop" investigation has been performed on the validity and accuracy of a new method of determining the reduction of the channel mobility connected to the increase of the longitudinal field. This method is applicable to standard transistors. An estimation of the saturation electron velocity in the channel has been accomplished comparing a widely used expression [1]. The data obtained with this procedure can be used to develop local models to account for the mobility dependence on the longitudinal-electric field in drift-diffusion simulators.

\section{DESCRIPTION OF THE METHOD}

The basic concept of idea of this method is the comparison of experimental drain current versus drain voltage curves $I_{D}-V_{D S}$, measured in shortchannel transistors to the results produced by a two-dimensional simulator $[2,3]$ in which an

\footnotetext{
* Corresponding author.
} 
accurate low-field mobility is used and the mobility reduction caused by the longitudinal-electric field is included. The total channel length $L$ is divided in $N$ subchannels with lengths $L_{i}, i=1,2, \ldots, N$, respectively. Instead of fixing the $L_{i}$ values, the channel potential values at the ends of the $i$-th subchannel, $V_{i-1}$ and $V_{i}$, are defined as the separation between the quasifermi levels at these points, $V_{0}=V_{S}$ and $V_{N}=V_{D}$ being the potentials at the total channel ends. Therefore, the separation into subchannels is obtained by dividing $V_{D}-V_{S}$ into very small intervals and fixing the channel potential at the ends of each subchannel. The number of subchannels is high enough and the difference $V_{i}-V_{i-1}$ in each subchannel is low enough that the longitudinal-electric field in each subchannel can be considered to be uniform. The application of the method is as follows:

1) Starting from a very small $V_{D S}$, for which one subchannel is enough, the gate-to-source voltage is varied and the low-field mobility as well as its dependence on the transverse-electric field is obtained.

2) $V_{D S}$ is increased in very small steps and the experimental result is compared to the simulated result calculated by using the low-field mobility obtained in point (1). New subchannels are introduced if necessary. When the experimental and computed currents deviate more than a prefixed (also very small) limit, $\delta I$, a mobility reduction is apparent at the subchannel placed at the drain end of the channel. Then, the mobility in this subchannel is multiplied by a factor of $\lambda<1$ in order to reproduce the experimental current again. The values for the transverse- and longitudinal-electric fields in this subchannel are introduced in an array: $E_{\text {trans }}(1)$ and $E_{\text {long }}(1)$, respectively.

3) $V_{D S}$ is increased again. In all the subchannels for which the longitudinal-electric field is greater than $E_{\text {long }}(1)$, the low-field mobility is multiplied by $\lambda$ and the current thus obtained is compared to the experimental one. When the deviation between the computed and experi- mental currents is again greater than $\delta I$, a further reduction is assumed in the drain end of the channel and the mobility is once more multiplied by $\lambda$, thus obtaining a value equal to the low-mobility multiplied by $\lambda^{2}$. The transverse- and longitudinal-electric fields in this subchannel are introduced as a new element of the array: $E_{\text {trans }}(2)$ and $E_{\text {long }}(2)$, respectively.

At the end of this process, the mobility values for a set of pairs ( $E_{\text {trans }}, E_{\text {long }}$ ) is obtained. This is schematized with the vertical solid line plotted in Figure 1.

\section{VALIDATION PROCEDURE}

Once we developed the algorithm to obtain the dependence of the mobility on the longitudinalelectric field we proceeded to validate it. To do so, we simulated several output curves, using our drift-diffusion simulator for a MOSFET and a supposed mobility dependence, after applying our method we were able to reproduce this dependence, confirming the correctness of the procedure. (Fig. 2).

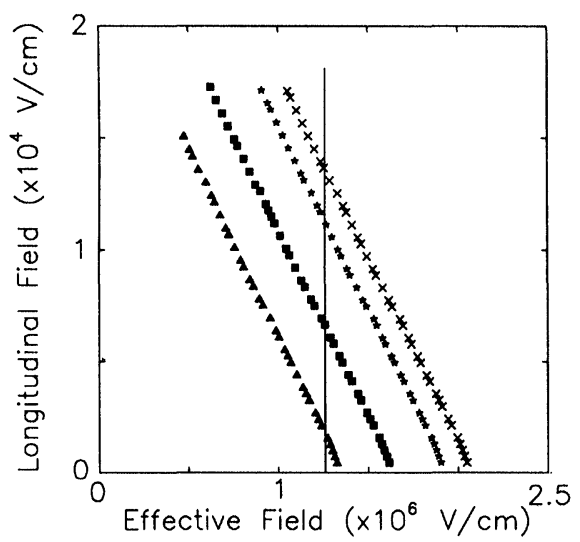

FIGURE 1 Tables $\left(E_{\operatorname{trans}(i)}, E_{\text {long }(i)}\right)$ used to account for the mobility reduction as the longitudinal field rises. They correspond to four different output curves. The solid line represents a common chosen effective field for all the tables. This value will be employed to obtain the mobility versus longitudinal field curves. 


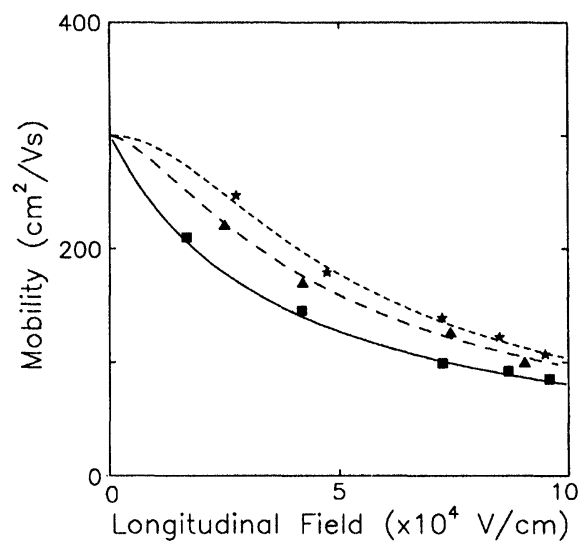

FIGURE 2 Mobility curves versus longitudinal-electric field obtained with Equation 1 to simulate output curves and validate this method. The $v_{\mathrm{SAT}}$ chosen was $v_{\mathrm{SAT}}=1.1 \times 10^{7}$ $\mathrm{cm} / \mathrm{s}, \beta=1$ (solid line), $\beta=1.5$ (long dashed line), $\beta=2$ (short dashed line). The results of our method are plotted in squares for $\beta=1$, triangles for $\beta=1.5$ and stars for $\beta=2$.

\section{EXPERIMENTAL RESULTS AND DISCUSSION}

After having validated the accuracy of our method, we applied it to Si MOSFETs fabricated by Lucent Technologies with $L_{\mathrm{EFF}}=0.6 \mu \mathrm{m}, W_{\mathrm{EFF}}$ $=20 \mu \mathrm{m}, T_{\mathrm{ox}}=157 \AA, N_{A}=1.2 \times 10^{17} \mathrm{~cm}^{-3}$ and $R_{\mathrm{SD}}=130 \Omega$. We have applied our technique to a set of measured output curves with $\delta I=10^{-3} \mathrm{~mA}$ and $\lambda=0.96$. The results obtained can be observed in Figure 3, where the mobility versus effective and longitudinal field surface is shown. Expression 1 was used to obtain the $\beta$ parameter and the saturation velocity $v_{\text {SAT }}$.

$$
V\left(E_{\|}, E_{\perp}\right)=\frac{\mu_{0}\left(E_{\perp}\right) E_{\|}}{\left(1+\left(\frac{\mu_{0}\left(E_{\perp}\right) \times E_{\|}}{v_{\text {sat }}}\right)^{\beta}\right)^{\frac{1}{\beta}}}
$$

The values that gave a better fit were $\beta=1$ and $v_{\text {SAT }}=1.1 \times 10^{7} \mathrm{~cm} / \mathrm{s}$. These values were independent of the effective field, confirming the result by Modelli and Manzini [4]. Using Thornber's expression with these parameters we have tried to

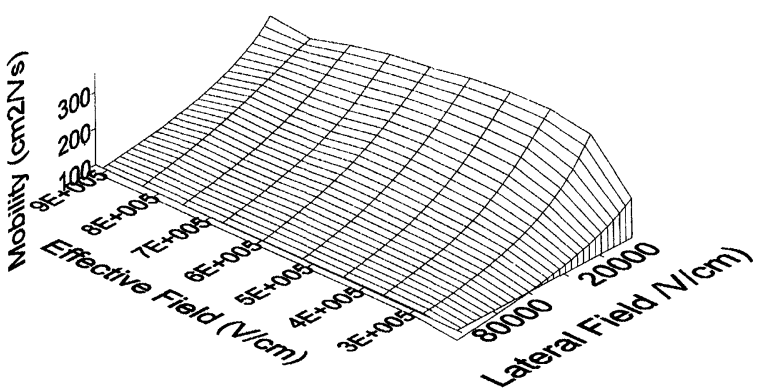

FIGURE 3 Experimental electron mobility as a function of the effective- and longitudinal-electric fields at room temperature.

reproduce the experimental output characteristics. The result is shown in Figure 4.

The data provided by this new procedure are very useful in order to obtain local mobility models for drift-diffusion simulators, where the dependence of the longitudinal-electric field is included. The influence of the series resistance and its bias dependence can be taken into account easily. It is also possible to assess the influence of electron velocity overshoot effects in very short channel MOSFETs by using a augmented mobility model [5].

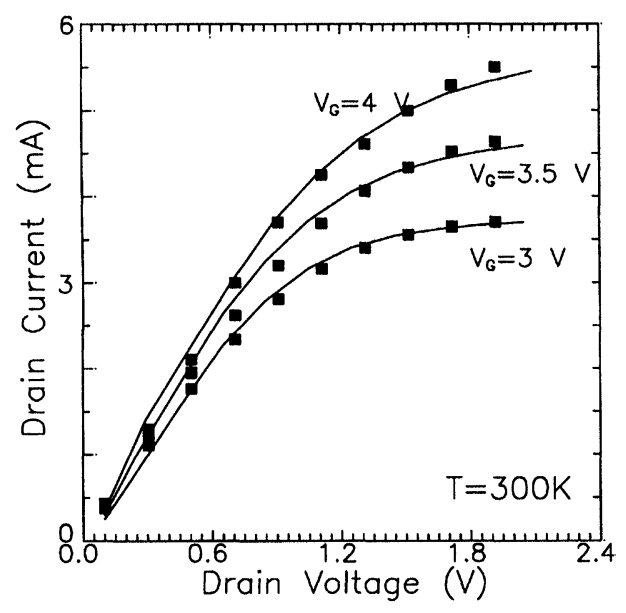

FIGURE 4 Experimental and simulated output curves at $T=$ $300 \mathrm{~K}$ for a $L_{\mathrm{EFF}}=0.6 \mu \mathrm{m}$ MOSFET. The simulated curves have been obtained by using the mobility shown in Figure 3, whose dependency on the longitudinal-electric field was obtained with this new method. 


\section{CONCLUSIONS}

A new experimental method for determining the dependence of the electron mobility on the longitudinal-electric field has been developed, validated and applied to standard submicron MOSFETs. This method can be easily implemented in a $2 \mathrm{D}$ drift-diffusion simulator. All the different dependencies of the electron mobility on the longitudinal-electric field can be taken into account at the same time by means of this method. A saturation-velocity value of $1.1 \times 10^{7} \mathrm{~cm} / \mathrm{s}$ was calculated by comparing the mobility curves to Thornber's expression.

\section{Acknowledgements}

We would like to thank José López Serrano from Lucent Technologies (Spain) for providing the MOSFETs $(0.9 \mu \mathrm{m}$ technology) we have used in our study. This work has been carried out within the framework of research project TIC 95-0511, supported by the Spanish Government (CICYT).

\section{References}

[1] Thornber, K. K. (1980). "Relation of drift velocity to lowfield mobility and high-field saturation velocity", J. Appl. Phys., 51, 2127.

[2] Roldán, J. B., Gámiz, F., López-Villanueva, J. A. and Carceller, J. E. (1995). "Monte Carlo simulation of a submicron MOSFET including inversion layer quantization", 4th IWCE, p. 37.

[3] Roldán, J. B., Gámiz, F., López-Villanueva, J. A. and Carceller, J. E. (1997). "Dependence of the electron mobility on the longitudinal electric field in MOSFETs", Semicond. Sci. Technol., 12, 321-330.

[4] Modelli, A. and Manzini, S. (1988). "High-field drift velocity of electrons in silicon inversion layers", Solid-State Electron., 31, 99.

[5] Roldán, J. B., Gámiz, F., López-Villanueva, J. A. and Carceller, J. E. (1997). "Modeling effects of electron velocity overshoot in a MOSFET", IEEE Transc. on Electron Devices, 44, 841-846.

\section{Authors' Biographies}

Juan B. Roldán graduated with a degree in physics in 1993, and received the Ph.D. in 1997 from the University of Granada. Since 1993 he has been working on the MOS device physics including 2D transport, non-local effects and Monte Carlo simulations. Current interests are also related to SiGe and SiC devices. He is a Teaching Assistant at the University of Granada.

Francisco Gámiz graduated with a degree in physics in 1991, and received the Ph.D. in 1994 from the University of Granada. Since 1991 he has been working on the characterization of scattering mechanisms and their influence on the transport properties of charge carriers in semiconductor heterostructures. His current research interest includes the effects of many-carriers on the electron mobility and the interpretation of the influence of high longitudinal electric fields have on MOS transistors. Current interest are also related to $\mathrm{SiGe}$ and $\mathrm{SiC}$, and $\mathrm{SCI}$ devices, and quantum transport. He has coauthored several papers in all these subjects. $\mathrm{He}$ is an Associate Professor at the University of Granada.

Juan A. López-villanueva graduated in 1984, Ph.D. in 1990 (University of Granada) with a thesis on the degradation of MOS structures by Fowler-Nordheim tunneling. Since 1985 he has been working on deep-level characterization and, mainly, MOS device physics, including FowlerNordheim and direct tunneling, quantum effects, 2D transport, effects of nonparabolicity, scattering mechanisms and Monte Carlo simulation of charge transport. He has coauthored several papers in all these subjects. His current research interest includes, simulation and modelling of electron devices. His educational activities also include analog systems for electronic instrumentation and power electronics. He is an Associate Professor at the University of Granada. 

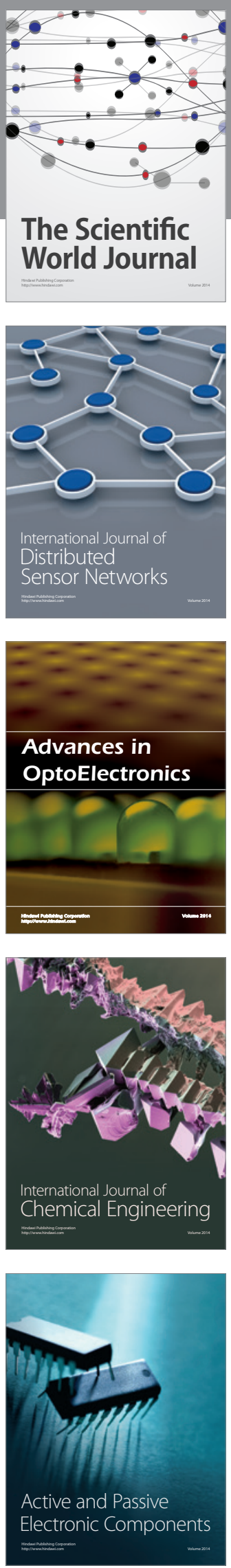
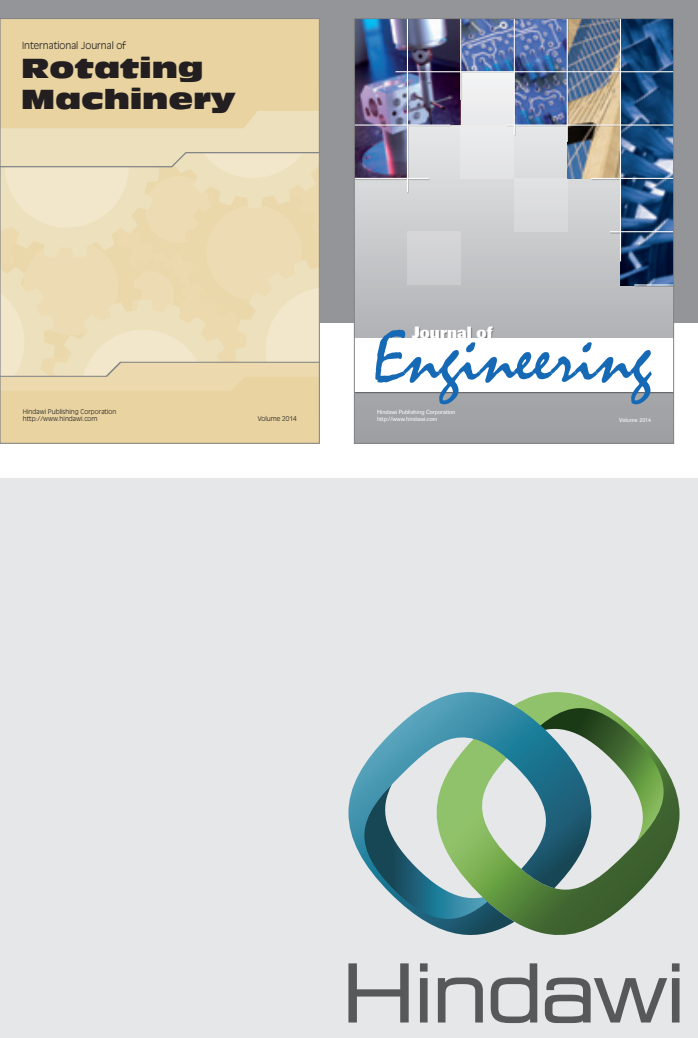

Submit your manuscripts at

http://www.hindawi.com
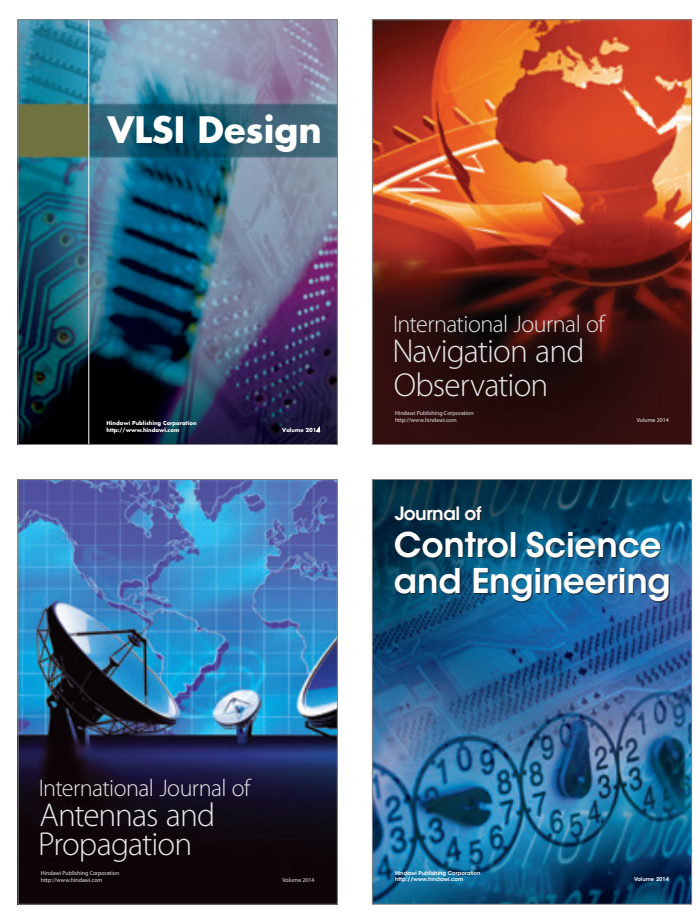
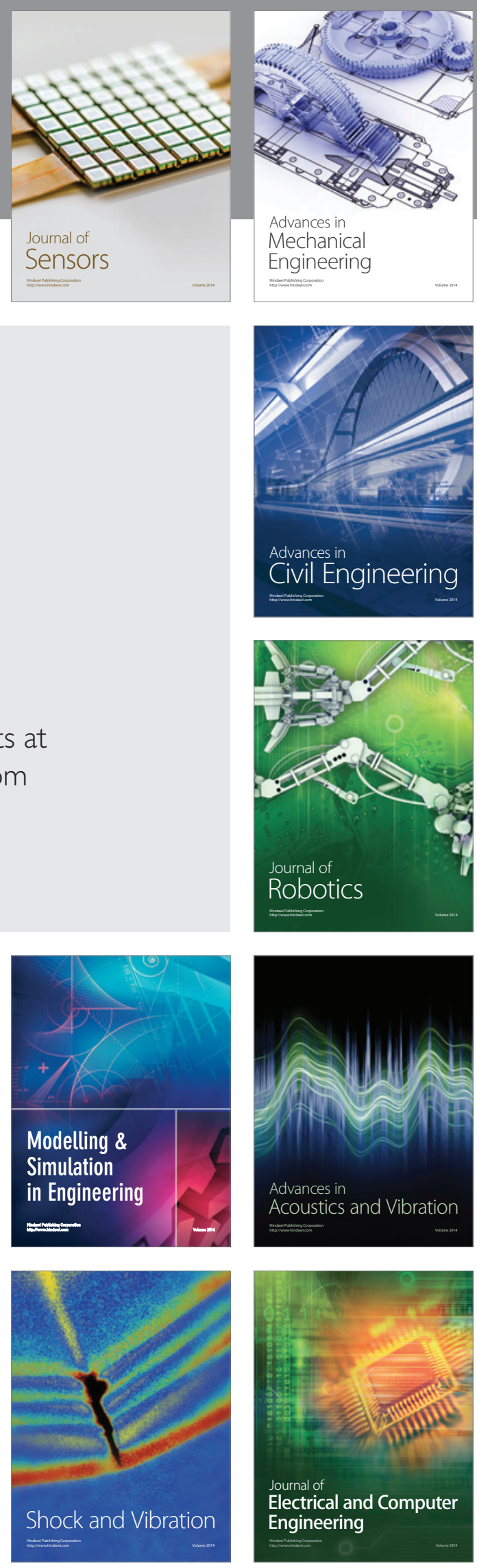